\title{
Hale: a multiwavelength far-infrared polarimeter for SOFIA
}

Charles Darren Dowell, J. A. Davidson, Jessie L. Dotson, Roger H. Hildebrand, Giles Novak, et al.

Charles Darren Dowell, J. A. Davidson, Jessie L. Dotson, Roger H.

Hildebrand, Giles Novak, Timothy S. Rennick, John E. Vaillancourt, "Hale: a multiwavelength far-infrared polarimeter for SOFIA," Proc. SPIE 4843,

Polarimetry in Astronomy, (14 February 2003); doi: 10.1117/12.458645

SPIE Event: Astronomical Telescopes and Instrumentation, 2002, Waikoloa, Hawai'i, United States 


\title{
Hale, a multi-wavelength, far-infrared polarimeter for SOFIA
}

\author{
C. D. Dowell ${ }^{a}$, J. A. Davidson ${ }^{b}$, J. L. Dotson ${ }^{c}$, R. H. Hildebrand ${ }^{d}$, \\ G. Novak ${ }^{e}$, T. S. Rennick ${ }^{f}$, and J. E. Vaillancourt ${ }^{g}$ \\ ${ }^{a}$ Department of Physics, California Institute of Technology \\ ${ }^{b}$ SOFIA Science Center, Universities Space Research Association \\ ${ }^{c}$ NASA-Ames Research Center \\ ${ }^{d}$ Department of Astronomy and Astrophysics, Department of Physics, and \\ Enrico Fermi Institute, University of Chicago \\ ${ }^{e}$ Department of Physics and Astronomy, Northwestern University

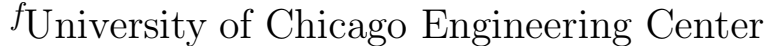 \\ ${ }^{g}$ Department of Physics, University of Wisconsin - Madison
}

\begin{abstract}
A far-infrared polarimeter, Hale, will be proposed for the next round of instruments for SOFIA. Key features are: simultaneous detection of two components of polarization; detector arrays providing $>4000$ pixels on the sky; and four passbands between $53 \mu \mathrm{m}$ and $215 \mu \mathrm{m}$, a range characterized by strong dependence of polarization on wavelength. At $53 \mu \mathrm{m}$ the diffraction-limited resolution, $1.2 \lambda / D$, will be 5.2 arcsec. In all passbands the systematic errors in polarization will be $\Delta P<0.2 \%, \Delta \theta<2^{\circ}$.
\end{abstract}

Keywords: polarimetry, far-infrared, instrumentation, bolometers, magnetic fields, SOFIA

\section{SCIENTIFIC JUSTIFICATION}

Observations with the polarimeters Stokes ${ }^{1}$ on the Kuiper Airborne Observatory and Hertz ${ }^{2,3}$ on the Caltech Submillimeter Observatory have shown that the far-infrared emission from molecular clouds is measurably polarized at almost every point. It is now possible to map the polarization, and hence to infer the shape of the projected magnetic field, at hundreds of points in individual clouds. ${ }^{4-6}$

\subsection{Magnetic pinches}

Figures 1 and 2 show polarization maps for the giant molecular clouds Orion and DR21. These maps, as recently extended, are presented here for the first time. The measured B-vectors and flux contours are shown together with B-field "profiles" from an empirical model adjusted to give an approximate fit to the Orion B-vectors (excluding the region near the "bar" in the southern portion of the map). The model corresponds to a symmetrical "hour-glass" magnetic pinch and the profiles are obtained from the model field lines by projecting onto the plane of the sky and averaging along the line of sight. ${ }^{7,8}$

Such pinched B-field profiles as given in Figs. 1 and 2 are expected for the case of the rapid supercritical collapse associated with star formation in a bound high-mass cluster ${ }^{9}$ when the axis of the field is near the plane of the sky. In Ref. 9 it is argued that the spatial extent of the supercritically collapsing region should be of order $0.3 \mathrm{pc}$ to $5 \mathrm{pc}$, which is the size of what the authors refer to as a "molecular clump". This dimension may be compared with the sizes of the regions in Orion and DR21 that contain the strongly pinched B-vectors. We estimate the sizes of these pinched-field regions by identifying the two corresponding B-field profiles (one on either side of the central density peak) that show the greatest deflection, and then taking the distance between these two curves at their closest approach. Using distances for Orion and DR21 of 500 pc and 2000

Author E-mail address:

C.D.D.: cdd@submm.caltech.edu 


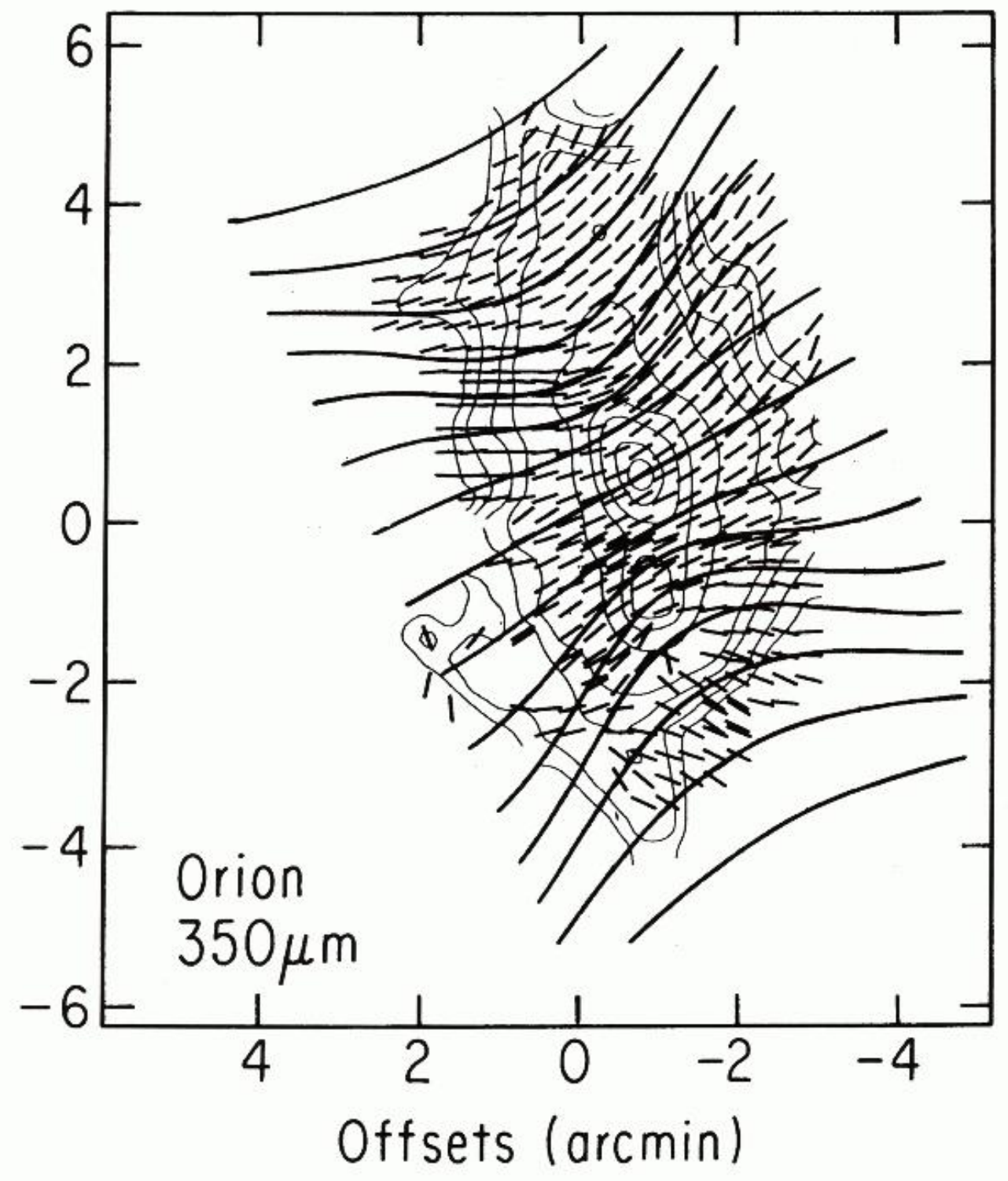

Figure 1. Magnetic "pinch" in Orion. The flux contours and polarization vectors are from observations with Hertz at the CSO. The "B-vectors" shown in the figure (measured E-vectors + 90 degrees), all of $\geq 3$ sigma significance, are drawn to a uniform length. Also shown are B-field "profiles" from the Orion pinch model of R. Rao. ${ }^{7}$ 


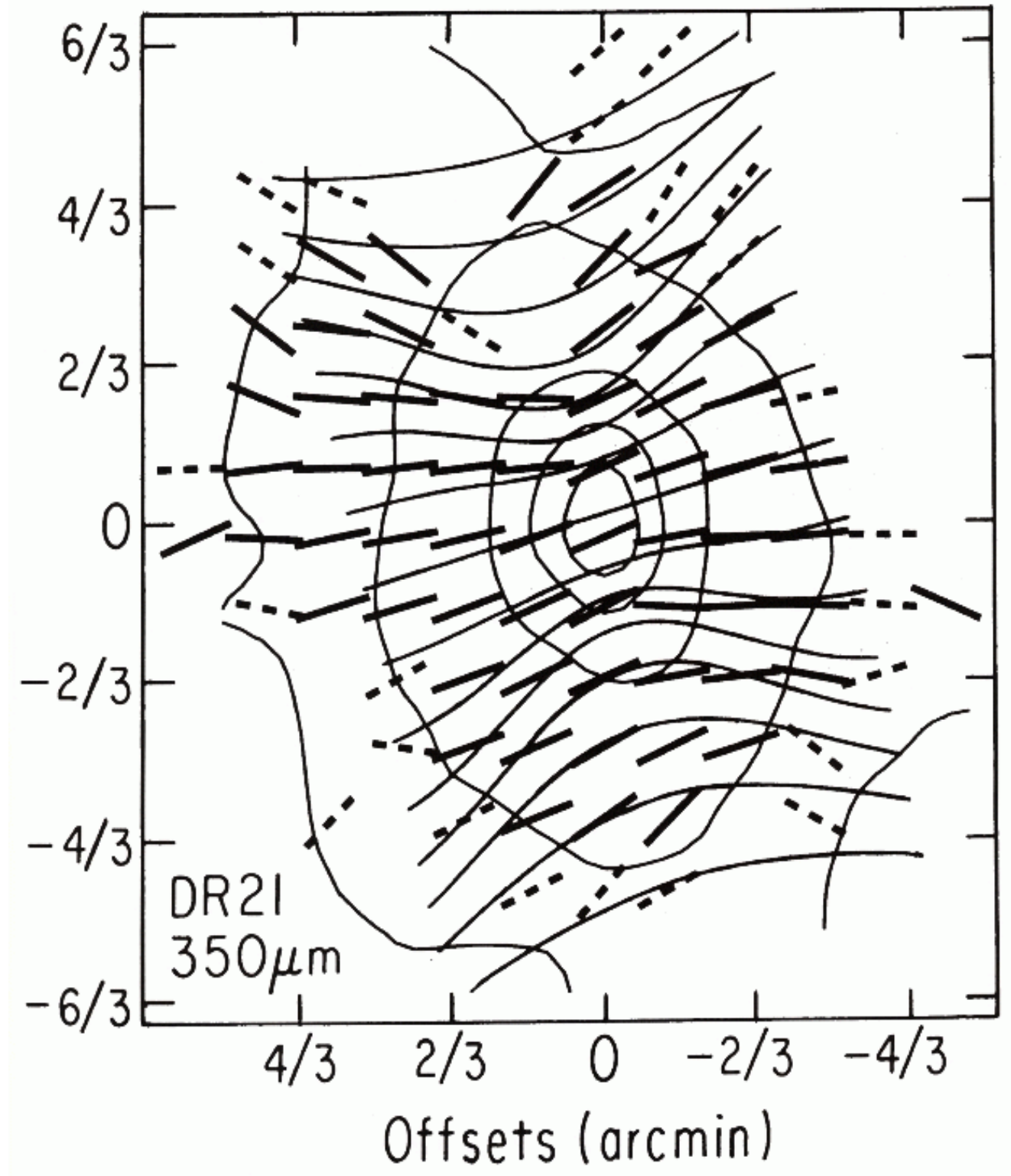

Figure 2. Magnetic "pinch" in DR21. As in Figure 1, the flux contours and B-vectors are from observations with Hertz at the CSO. The solid vectors indicate $\geq 3$ sigma significance; the dashed vectors, 2 to 3 sigma significance. The pinch model is the same as for Orion but reduced in angular scale by a factor of three, to correspond to the greater distance to DR21. The 3-fold reduction is a compromise: the ratio of the distances is actually closer to four, but the linear width of DR21 is significantly greater than that of Orion. 

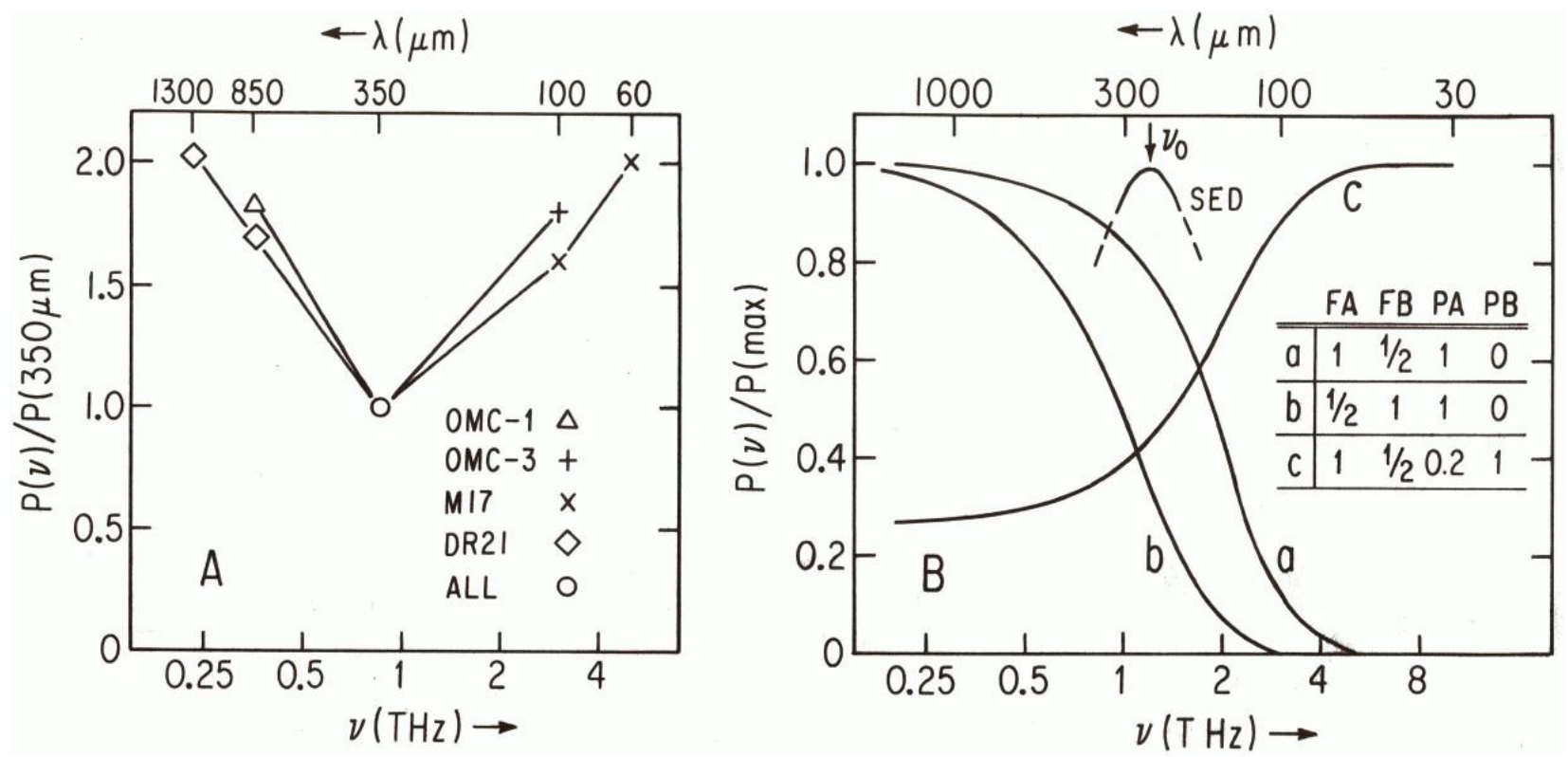

Figure 3. Polarization spectra. A) Measured spectra ${ }^{12}$ for several molecular clouds (normalized at $350 \mu m$ ). B) Computed spectra for three models of the diffuse emission assuming two components, $A$ and $B$, with relative flux peaks $F A, F B$, temperatures $T A, T B$, polarizing powers $P A, P B$, and spectral indexes $\beta A, \beta B$. The curves are drawn for $\Delta T=6 K \approx$ value given by Ref. 14 .

pc, respectively, we find that the spatial extent of the observed pinches is $\sim 0.7 \mathrm{pc}$ for both sources, in fair agreement with the range of expected pinch sizes given above.

There is compelling evidence, beyond that presented here, to support the idea that high mass star formation occurs via supercritical collapse on scales of $\sim 1$ pc. ${ }^{9}$ By contrast the mechanism of low mass star formation is still open to question. The conventional picture is that there must be a long period during which the field slowly diffuses out of dense subcritical cores, followed by a supercritical collapse on scales much smaller than for high mass star formation. However, the recent detection of relatively high collapse velocities in regions where low mass stars are forming has raised doubts about the relevance of ambipolar diffusion and magnetic subcriticality. ${ }^{10}$ With its superior sensitivity and angular resolution, Hale on SOFIA will be able to map relatively faint nearby clouds at linear scales far below those indicated in Figures 1 and 2. The scale of any pinches discovered in low mass star forming regions will be important for testing theoretical models.

\subsection{Polarization spectra}

Other investigations that will be possible with Hale's capabilities include magnetic fields in disks around young stellar objects, characterization of the polarized cosmic foreground, and characterization of the turbulent components of magnetic fields in dense clouds.

Until 1995, our group observed polarization only at $100 \mu \mathrm{m}$. For the last flight series with Stokes before the $\mathrm{KAO}$ was decommissioned, we shifted the passband to $60 \mu \mathrm{m} .{ }^{11}$ At about the same time we began operating a $350 \mu \mathrm{m}$ polarimeter, Hertz, at the Caltech Submillimeter Observatory. When the results at 60, 100, and $350 \mu \mathrm{m}$ were compared, and later when comparisons could be made with results at $850 \mu \mathrm{m}$ from the SCUBA polarimeter we discovered that the degree of polarization depended strongly on wavelength. ${ }^{12,13}$ We have interpreted this as an effect of differences in the environments of dust grains in cloud components at different temperatures. Accordingly we expect contrasting polarization spectra for different types of objects such as molecular clouds, infrared cirrus, and dark clouds. Figure 3A shows measured polarization spectra for several giant molecular clouds. 
Cosmologists measuring polarized emission from the early universe require accurate models of the polarized foreground emission. Broad surveys including faint regions of the diffuse interstellar medium will require polarimeters on balloon and space platforms. Hale on SOFIA, however, will be uniquely qualified to measure the far-IR polarization spectrum of the diffuse emission in regions that are relatively bright but sufficiently tenuous so that all grains are exposed to the same radiation field. There are scores of diffuse regions with flux densities in the range $10-100 \mathrm{MJy} / \mathrm{sr}\left(0.024-0.24 \mathrm{Jy}\right.$ per $\left.\left(10 \times 10 \operatorname{arcsec}^{2}\right)\right)$, which is more than adequate for Hale polarimetry: blocks of pixels, say $10 \times 10$, can be combined while still exceeding the resolution of any currently planned cosmological observations of polarized emission. The tenuous character of the clouds can be determined by their spectral energy distributions (steep spectra from $100 \mu \mathrm{m}$ to $60 \mu \mathrm{m}$ and flat spectra from $60 \mu \mathrm{m}$ to $25 \mu \mathrm{m}$ ). Because all grains in these clouds are in the same environment, their polarization spectra should be much simpler to analyze than those of molecular clouds. Figure 3B shows computed polarization spectra according to three models. Assuming a two-component model as inferred from COBE data, ${ }^{14}$ one can solve for the temperatures, peak fluxes, spectral indexes and polarizing powers of the components from measurements of the slopes and asymptotes of the curves, from their positions with respect to the peak of the spectral energy distribution, and from the FWHM of the spectral energy distribution.

\subsection{Turbulence}

In the diffuse medium, the amplitude and scale of turbulent domains have been inferred from data on the rate of increase with column density, $N$, of the polarization, $P$, of starlight. ${ }^{15,16}$ In dense clouds, an analogous investigation, favored by SOFIA's high angular resolution, can be used to infer the properties of the turbulent component from the rate of decrease of $P$ with $N$ and from measurements of angular dispersion vs $N$. Here the analysis is complicated by the dependence of $P$ on environmental conditions along the line of sight and the dependence of those conditions on $N$. Nevertheless there is a strong incentive, and now a strong effort, to work through the complications so that the turbulent component of the field can be characterized and compared with velocity dispersion in various classes of objects.

Hale will be able to resolve the small polarization domains thus far observed only in a restricted region of Orion's core by millimeter-wave interferometry. ${ }^{17}$ Hale will map with comparable resolution over a much wider area and, because it will be sensitive to the warm dust in the core, it will discriminate against emission from cool foreground and background dust. The results should settle whether these are turbulent domains in the magnetic field or domains in which grain alignment is dominated by local outflows.

\section{INSTRUMENT DESIGN}

To exploit SOFIA's potential for far-infrared polarimetry Hale must 1) Provide multiple passbands distributed over a wide range of wavelengths; 2) Take full advantage of SOFIA's superior angular resolution in the farinfrared; 3) To the extent possible, fill SOFIA's useful focal plane with diffraction-limited and backgroundlimited detectors; and 4) Minimize the effects of atmospheric noise on polarization measurements.

Hale will have four passbands in the range $53 \mu \mathrm{m}$ to $215 \mu \mathrm{m}$ with provision for a fifth band (§2.1). Throughout this range, SOFIA will provide the best spatial resolution available for polarimetry. At $53 \mu m$, the diffractionlimited resolution, $1.2 \lambda / D$, will be 5.2 arcsec., a value four times better than that attained by any previous far-infrared polarimetry.

To fill SOFIA's useful focal plane with pixels of diameter $\lambda / 2 D$ it will be necessary to increase the number of pixels by more than an order of magnitude beyond that of any first-round far-IR instrument for SOFIA, and more than two orders of magnitude over that used in any previous airborne polarimetry. At the time of this symposium it appears that it will be feasible to construct arrays of $64 \times 64(=4096)$ pixels by the time the design for Hale must be fixed ( 2005).

At SOFIA's cruising altitude, normally $\geq 41,000 \mathrm{ft}(12.5 \mathrm{~km})$, fluctuations in atmospheric emission are comparable in magnitude to the polarized flux from sources of moderate intensity. The effects of these fluctuations on measurements of polarization are reduced by more than an order of magnitude by simultaneous detection of two components of polarization. ${ }^{3,6}$ It is therefore an essential feature of the design to incorporate a polarizing beam splitter and to provide a detector array for each component. A further reduction in the effects of these 


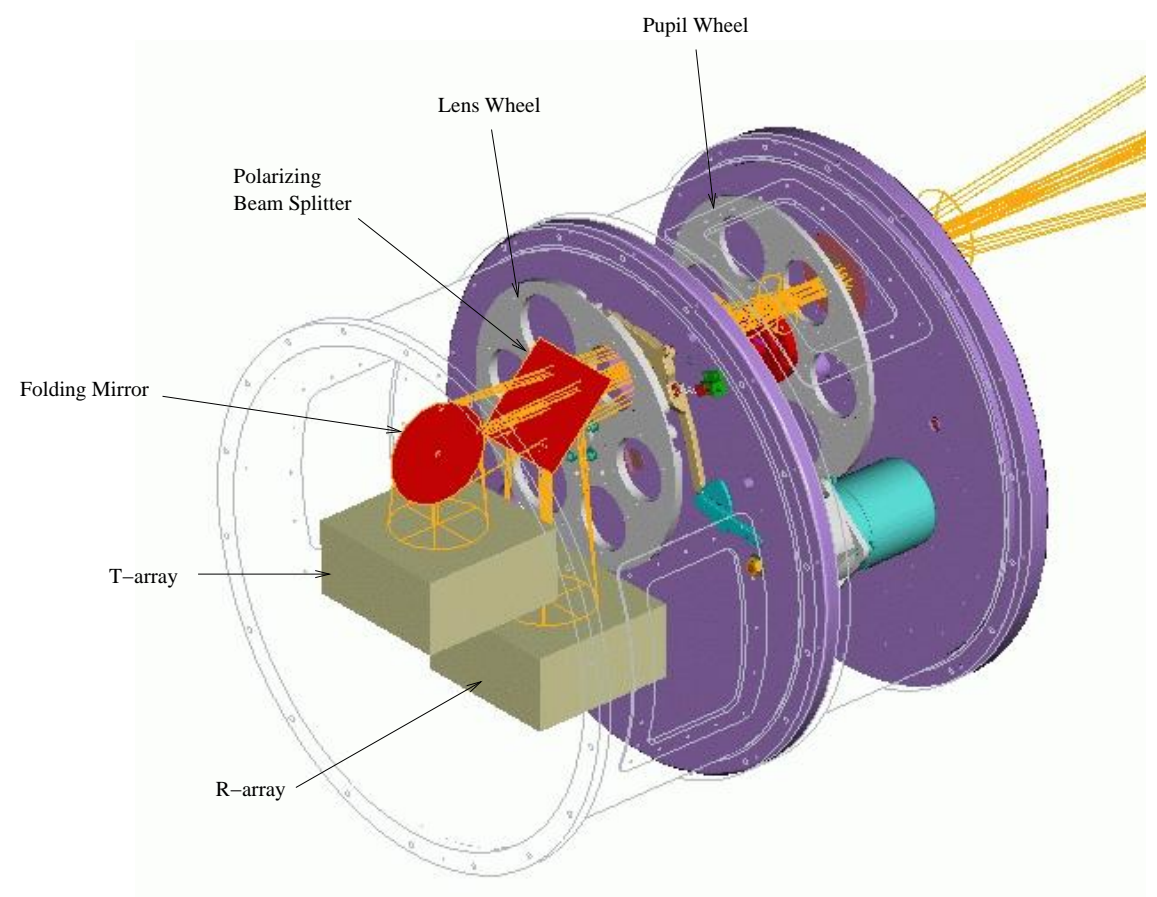

Figure 4. Schematic diagram showing basic concept of Hale's optical design.

fluctuations will be achieved by careful selection of passbands to avoid frequencies of relatively high atmospheric absorption and emission (see $\S 2.1$ ).

As a design philosophy, Hale will adopt as much as possible the mechanical design of HAWC, ${ }^{18}$ the first-light, far-infrared camera for SOFIA, in order to reduce engineering and airworthiness certification costs.

\subsection{Choice of Passbands}

To first approximation, one would like to space the passbands at equal intervals in $\log \lambda$. But the effects of atmospheric fluctuations can be substantially reduced by selecting passbands that minimize absorption and emission, a strategy largely ignored in previous airborne astronomy. The adjustment of bandwidths can also be used to equalize loading of the detectors from one band to the next. The choice of bands to balance these factors has been carefully considered in the design of HAWC. We plan, initially, to adopt the HAWC passbands (tentatively 53, 88, 155, and $215 \mu \mathrm{m}$ ) not only for the technical reasons just mentioned, but also to facilitate comparisons of complementary results from the two instruments. Our design, however, permits easy shifts to other passbands as may be required for particular scientific objectives. We also provide a fifth "band" which can be used to fill in another wavelength or to provide a lower image magnification so as to increase the area coverage.

\subsection{Optics}

The Hale optical system, incorporating aspects of the NASA/GSFC-designed optomechanical system for HAWC, is shown in Figure 4. The principal components are: fore-optics ( $\$ 2.2 .1$; not shown in figure); a half-wave plate module mounted on the pupil wheel and holding a spectral filter, half-wave plate, actuator, encoder, and lenses to produce an image of the sky of the desired magnification at the detector arrays; a polarizing beam splitter; and two detector arrays, one for each of two components of polarization. 


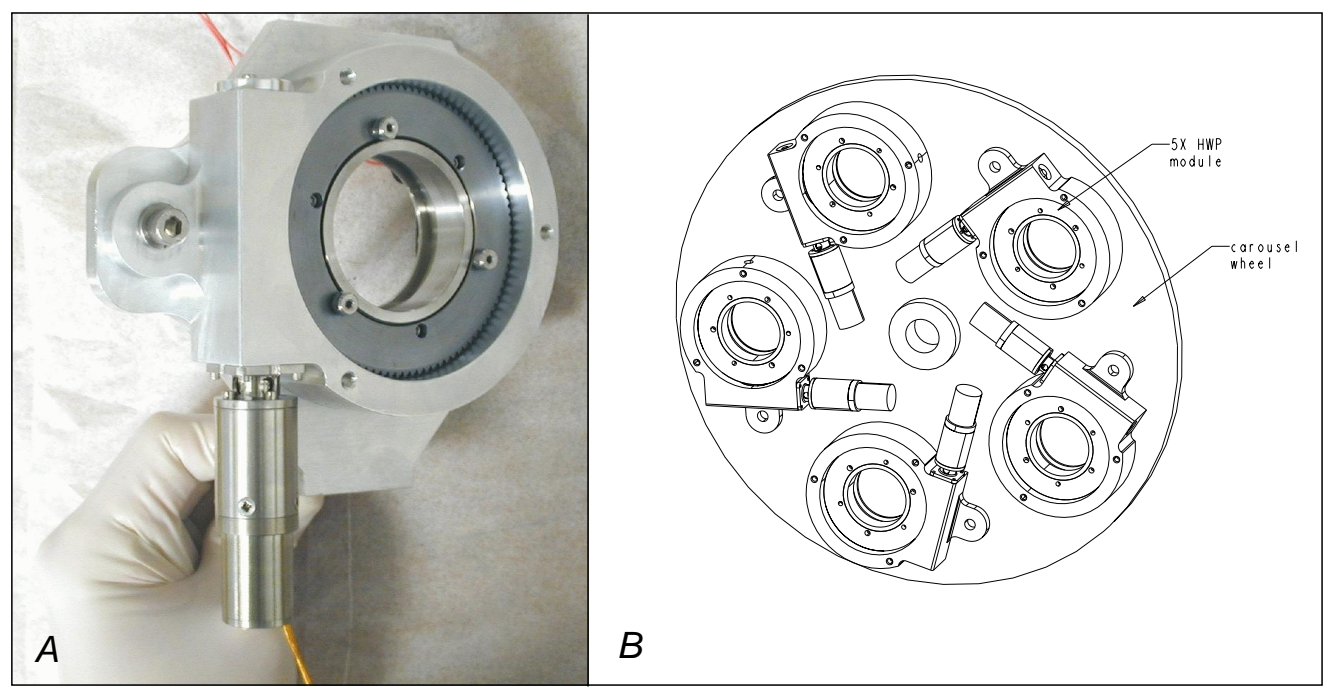

Figure 5. A) Prototype half-wave plate module for Hale. ${ }^{19}$ B) Concept drawing of pupil wheel with five half-wave plate modules.

\subsubsection{Fore-optics}

The largest source of emission in the instrument optical path is the vacuum window of the cryostat. The required diameter, and hence thickness of that window can be greatly reduced by introducing a folding flat and field mirror ahead of the cryostat so that the instrument pupil falls just inside the window. The thermal emission from the warm fore-optics is more than offset by the reduced emission from the window. Tests of a prototype $0.8 \mathrm{~mm}$ window made of high-density polyethylene have shown that the $8 \mathrm{~cm}$ diameter clear aperture required by the optics can safely be obtained. This is a somewhat thinner window, and therefore more transmissive, than was used for the $5 \mathrm{~cm}$ windows of photometers and polarimeters on the Kuiper Airborne Astronomy.

\subsubsection{Half-wave plate module}

By rotating a stage, any one of five half-wave plate modules can be inserted into the optical path. A prototype half-wave plate (Fig. 5A) driven by a cryogenic motor has been successfully tested in the laboratory. ${ }^{19}$ Five such modules will be mounted on a rotating stage (Fig. 5B), with motor leads running inside a hollow central shaft on the pupil wheel.

\subsubsection{Polarizing beam splitter}

As we used for the $60 \mu \mathrm{m}$ configuration of Stokes, ${ }^{11}$ the polarizing beam splitter for Hale will consist of fine grids of parallel conductors (period $\approx 1 / 5 \times$ minimum $\lambda$ ) deposited on mylar substrates by photolithography. The polarization modulation efficiency will be $>95 \%$ for all passbands.

\subsection{Detector arrays}

Our current design assumption, based on discussions at the USRA/SOFIA Detector Workshop held in April 2002 , is that the pixels in the arrays will be close-packed transition-edge sensors (TES) with dimension 1.5 mm $\times 1.5 \mathrm{~mm}$ and that a $64 \times 64$ pixel array will therefore cover an area of $\sim 10 \mathrm{~cm} \times 10 \mathrm{~cm}(\mathrm{or} \sim 15 \mathrm{~cm} \times 15 \mathrm{~cm}$ including the surrounding frame). We estimate a depth of $7 \mathrm{~cm}$ to accommodate the readout electronics. 


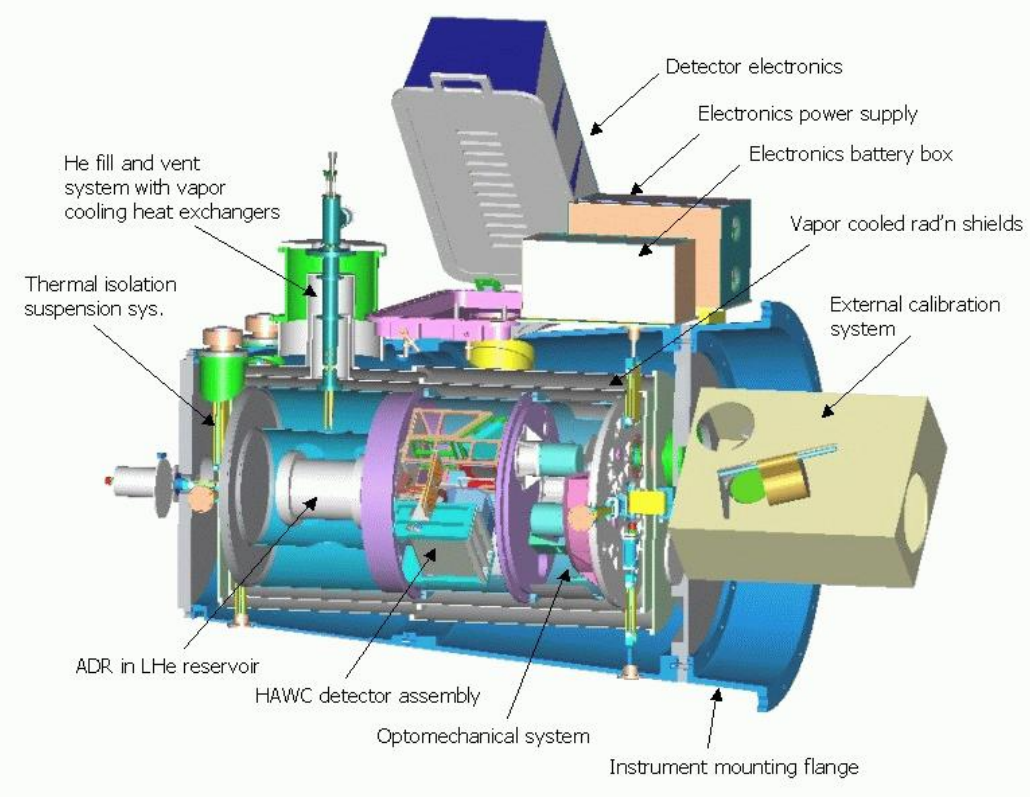

Figure 6. Cut-away view of HAWC, ${ }^{18}$ a first light SOFIA instrument now in an early stage of construction. Hale will adopt HAWC's fore-optics and cryostat designs.

The relatively small volume required by the multiplexed readout electronics for Hale's TES detectors makes it possible to fit these two arrays into the volume required by the conventional readout electronics for HAWC's single 12 x 32 - pixel array of semiconductor sensors.

\subsection{Cryostat}

We will save considerably in cost and time by adopting the HAWC cryostat design. That design (Fig. 6) will meet all Federal Aviation Administration air-worthiness requirements for the SOFIA Observatory and is currently in the early stages of fabrication.

\subsection{Specifications}

The specifications for Hale are summarized in Table 1. A fifth band, not shown in the table, can be used for observations at an additional wavelength and/or a different magnification.

\section{ACKNOWLEDGMENTS}

We thank S. Wang for work on the optical design shown in Fig. 4, and D. Chuss for help in preparing Table 1 and Fig. 4. The observations with Hertz and the development of the half-wave plate module were supported by NSF grants AST 9987441 and AST 0204886.

\section{REFERENCES}

1. S. R. Platt, R. H. Hildebrand, R. J. Pernic, J. A. Davidson, \& G. Novak, "100- $\mu m$ Array Polarimetry from the Kuiper Airborne Observatory: Instrumentation, Techniques, and First Results," PASP, 103, 1193, 1991

2. D. A. Schleuning, C. D. Dowell, R. H. Hildebrand, S. R. Platt, \& G. Novak, "Hertz, a Submillimeter Polarimeter," PASP, 109, 307, 1997 
Table 1. Hale Specifications

\begin{tabular}{lllll}
\hline \hline Parameter & Band 1 & Band 2 & Band 3 & Band 4 \\
\hline Central wavelength $(\mu m)$ & 53 & 88 & 155 & 215 \\
Central frequency $(\mathrm{THz})$ & 5.7 & 3.4 & 1.9 & 1.4 \\
Band width, FWHM $(\Delta \lambda / \lambda)$ & 0.10 & 0.10 & 0.14 & 0.20 \\
\hline Pixel size (arcsec) & 2.25 & 3.5 & 6.0 & 8.0 \\
Pixel solid angle $\left(10^{-9}\right.$ sr) & 0.12 & 0.29 & 0.85 & 1.5 \\
Field of view: $64 \times 64$ array $($ arcmin $)$ & $2.4 \times 2.4$ & $3.7 \times 3.7$ & $6.4 \times 6.4$ & $8.5 \times 8.5$ \\
Resolution, $1.2 \lambda / D($ arcsec $)$ & 5.2 & 8.6 & 15.2 & 21 \\
\hline Background power/pixel $(\mathrm{pW})$ & 13 & 12 & 14 & 10 \\
NEFD $\left(\mathrm{Jy} \mathrm{s}^{1 / 2}\right)$ & 2.0 & 1.3 & 1.2 & 0.7 \\
\hline$\sigma(P), 1 \mathrm{hr} ., 30 \mathrm{Jy}$ source $(\%)$ & 0.21 & 0.14 & 0.13 & 0.083 \\
$\sigma(P), 10 \mathrm{hr} ., 1 \mathrm{Jy}$ source $(\%)$ & 2.1 & 1.4 & 1.3 & 0.79 \\
\hline$\sigma(\theta), 1 \mathrm{hr} ., 30$ Jy source, $P=3 \%$ & $2^{\circ}$ & $1^{\circ}$ & $1^{\circ}$ & $1^{\circ}$ \\
$\sigma(\theta), 10 \mathrm{hr} ., 1$ Jy source, $P=3 \%$ & $20^{\circ}$ & $13^{\circ}$ & $14^{\circ}$ & $7^{\circ}$ \\
\hline \hline
\end{tabular}

3. C. D. Dowell, R. H. Hildebrand, D. A. Schleuning, J. E. Vaillancourt, J. L. Dotson, G. Novak, T. Renbarger, \& M. Houde, "Submillimeter Array Polarimetry with Hertz," ApJ, 504, 588, 1998

4. J. L. Dotson, J. A. Davidson, C. D. Dowell, D. A. Schleuning, \& R. H. Hildebrand, "Far-Infrared Polarimetry of Galactic Clouds from the Kuiper Airborne Observatory," ApJS, 128, 335, 2000

5. R. H. Hildebrand, J. L. Dotson, C. D. Dowell, G. Novak, D. A. Schleuning, \& J. E. Vaillancourt, "Hertz, an Imaging Polarimeter," in SPIE Conf. on Advanced Technology MMW, Radio, and Terahertz Telescopes, SPIE Vol. 3357, 289, 1998

6. R. H. Hildebrand, J. A. Davidson, J. L. Dotson, C. D. Dowell, G. Novak, \& J. E. Vaillancourt, "A Primer on Far-Infrared Polarimetry," PASP, 112, 1215, 2000. Erratum: PASP, 112, 1620, 2000

7. R. Rao, private communication, 2002

8. D. A. Schleuning, "Far-Infrared and Submillimeter Polarization of OMC-1: Evidence for Magnetically Regulated Star Formation," ApJ, 493, 811, 1998

9. F. H. Shu, F. C. Adams, \& S. Lizano, "Star Formation in Molecular Clouds: Observation and Theory," Ann. Rev. Astron. Astrophys., 25, 23, 1987

10. N. J. Evans, II, "Physical Conditions in Regions of Star Formation," Ann. Rev. Astron. Astrophys., 37, 311,1999

11. C. D. Dowell, "Far-Infrared Polarization by Absorption in the Molecular Cloud Sagittarius B2," ApJ, 487, 237,1997

12. J. E. Vaillancourt, "The Far-Infrared/Submillimeter Polarization Spectrum and Temperature Maps of Molecular Clouds," ApJS, in press, 2002

13. R. H. Hildebrand, J. L. Dotson, C. D. Dowell, D. A. Schleuning, \& J. E. Vaillancourt, "The Far-Infrared Polarization Spectrum: First Results and Analysis," ApJ, 516, 834, 1999

14. D. P. Finkbeiner, M. Davis, \& D. J. Schlegel, "Extrapolation of Galactic Dust Emission at 100 Microns to Cosmic Microwave Background Radiation Frequencies Using FIRAS," ApJ, 524, 867, 1999

15. T. J. Jones, D. Klebe, \& J. M. Dickey, "Infrared Polarimetry and the Galactic Magnetic Field II. Improved Models," ApJ, 389, 602, 1992

16. P. Fosalba, A. Lazarian, S. Prunet, \& J. A. Tauber, "Statistical Properties of Galactic Starlight Polarization," ApJ, 564, 762, 2002

17. R. Rao, R. M. Crutcher, R. L. Plambeck, \& M. H. C. Wright, "High Resolution Millimeter-Wave Mapping of Linearly Polarized Dust Emission: Magnetic Field Structure in Orion," ApJ, 502, L75, 1998 
18. D. A. Harper, C. A. Allen, M. J. Amato, T. Ames, A. Bartels, S. C. Casey, R. J. Derro, Rh. Evans, I. Gatley, S. J. Heimsath, A. Hermida, M. D. Jhabvala, J. H. Kastner, R. F. Loewenstein, S. H. Moseley, R. J. Pernic, T. Rennick, H. E. Rhody, D. Sandford, R. A. Shafer, P. J. Shirron, G. Voellmer, S. Wang, \& J. Wirth, "HAWC: A Far-Infrared Camera for SOFIA," SPIE Conf. Series Vol. 4014, Airborne Telescope Systems, Eds. R. K. Melugin \& H. Roeser, 2000

19. T. S. Rennick, J. E. Vaillancourt, R. H. Hildebrand, \& S. J. Heimsath, "A Cryogenic Half-Wave Plate Module to Measure Polarization at Multiple FIR Passbands," Proc. 36th Aerospace Mechanisms Symposium, Glenn Research Center, in press, 2002 